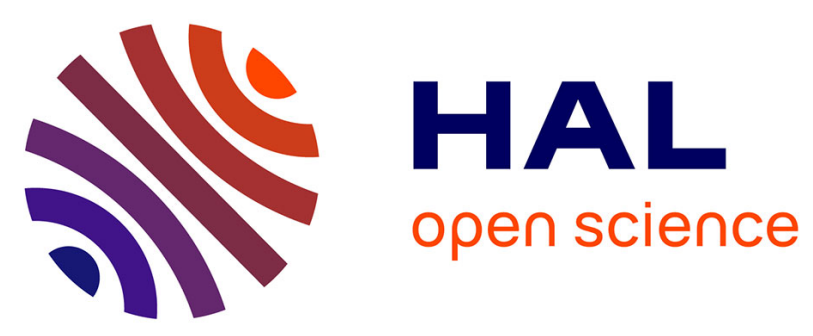

\title{
Dielectric analysis of water uptake in polymer coating using spatially defined Fick's law and mixing rule
}

\author{
Lucas Ollivier-Lamarque, Mickaël Null Lallart, Nicolas Mary, Tetsuya
}

Uchimoto, Sébastien Livi, Sabrina Marcelin, Hiroyuki Miki

\section{- To cite this version:}

Lucas Ollivier-Lamarque, Mickaël Null Lallart, Nicolas Mary, Tetsuya Uchimoto, Sébastien Livi, et al.. Dielectric analysis of water uptake in polymer coating using spatially defined Fick's law and mixing rule. Progress in Organic Coatings, 2020, 148, pp.105846. 10.1016/j.porgcoat.2020.105846 . hal-02971027

\section{HAL Id: hal-02971027 \\ https://hal.science/hal-02971027}

Submitted on 22 Oct 2020

HAL is a multi-disciplinary open access archive for the deposit and dissemination of scientific research documents, whether they are published or not. The documents may come from teaching and research institutions in France or abroad, or from public or private research centers.
L'archive ouverte pluridisciplinaire HAL, est destinée au dépôt et à la diffusion de documents scientifiques de niveau recherche, publiés ou non, émanant des établissements d'enseignement et de recherche français ou étrangers, des laboratoires publics ou privés. 


\title{
Dielectric Analysis of Water Uptake in Polymer Coating using Spatially Defined Fick's Law and Mixing Rule
}

\author{
Lucas OLLIVIER-LAMARQUE ${ }^{\mathrm{a}, \mathrm{b}, \mathrm{c}}$, Mickaël LALLART ${ }^{\mathrm{c}, \mathrm{d}, \mathrm{a}, *}$, Nicolas MARY $^{\mathrm{a}, \mathrm{e}}$, Tetsuya \\ UCHIMOTO $^{\mathrm{c}, \mathrm{a}}$, Sébastien LIVI $^{\mathrm{f}}$, Sabrina MARCELIN ${ }^{\mathrm{e}}$, Hiroyuki MIKIc \\ ${ }^{a}$ ELyTMaX UMI 3757, CNRS - Université de Lyon - Tohoku University, International Joint Unit, \\ Tohoku University, Sendai, Japan \\ ${ }^{b}$ Graduate School of Engineering, Tohoku University, Sendai, Japan \\ ${ }^{c}$ Institute of Fluid Science, Tohoku University, Sendai, Japan \\ ${ }^{d}$ Univ. Lyon, INSA-Lyon, LGEF EA 682, F-69621, France \\ e Univ. Lyon, INSA-Lyon, MATEIS UMR CNRS 5510, F-69621, France \\ ${ }^{f}$ Univ. Lyon, INSA-Lyon, IMP UMR CNRS 5223, F-69621, France
}

\begin{abstract}
Due to their outstanding properties such as thermal insulation, optical transparency or adhesion for instance, thermosetting coatings based on epoxy prepolymers offer many attractive advantages. In the framework of investigating their ageing under wet conditions, this study exposes an original approach taking into account the water penetration depth. This allows the derivation of local permittivity using mixing rule, ultimately yielding a physically-based explanation of the globally measured capacitance of immersed polymer coupons placed between circular electrodes and its link to gravimetric results. Theoretical predictions show good agreement with experimental measurements on epoxy-amine coupons both in terms of mass uptake and dielectric constants (including frequency dependence), bringing new insights in uptake mechanisms and associated measurement techniques.
\end{abstract}

Keywords: polymer coating, thermosetting coating, water uptake, gravimetry, dielectric analysis

\section{Introduction}

The large use of metallic structures, for example in transportation systems (passengers, goods, electricity, gas...), infrastructures, manufacturing, machining and so on, raises challenges in sustainability and reliability of associated systems, especially when those are operating in confined or

5 barely accessible environments (e.g., pipelines). Among the damaging processes, corrosion is one of the major stakes such structures are facing ([1]). Therefore, many works have been devoted in finding adapted techniques to prevent corrosion, including structural transformation, special treatment or coating $([2,3])$, along with the development of new formulations. Regarding the latest solution, polymer $([4,5])$ and polymer composite ([6, 7] $)$ coating offers several advantages including significant barrier effect, low cost, simple processing, insulating properties and high strength

\footnotetext{
${ }^{*}$ Corresponding author

Email address: mickael.lallart@insa-lyon.fr (Mickaël LALLART)
} 
to weight ratio. However, thermosetting coating, especially epoxy resin-based ones that are widely used, are sensitive to water uptake that degrades the coating performance eventually compromising the protection sought from their application ([8, 9]).

Hence, being able to monitor and predict the structural integrity of these kinds of coating layer represents significant importance to assess the risk of damage of the protected structure. In addition to the evaluation of conventional parameters such as coating thickness (for instance using Eddy Current - [10] -, ultrasonic testing - [11] -, microwave backscattering - [12] - or nearinfrared reflection spectroscopy - [13]) or adequate deposition ([14, 15]), water uptake evaluation is typically done using gravimetric approach, through the monitoring of the mass evolution due to 20 water absorption $([16,17)$. Nevertheless, coupon extraction for gravimetric measurements without damaging the operating structure (such as pipe for instance) is barely feasible. Hence, in order to be able to assess the integrity of the coating by in-situ techniques, dielectric analysis through direct capacitance measurements $([18,19,20])$ or by means of Electrochemical Impedance Spectroscopy (EIS - 21, 22]) for instance and its correlation with water mass uptake (23, 24]) has been proposed. ${ }_{25}$ While they are, at a first glance, not as precise as other advanced non destructive measurement techniques such as FTIR (Fourier-Transform Infrared Spectroscopy - 25, 26]), AFM-IR (Atomic Force Microscope Infrared-Spectroscopy - 27]) or sorption analysis (28]) in terms of water uptake detection, they are simple, low cost, and can give some quantitative information.

However, conventional gravimetry and dielectric analyses, including associated modeling as well as their correlations, are usually conducted considering global measurements. This therefore yields, in addition to some quantitative inconsistencies between the two approaches (29, 30]), to perfectible results in terms of coating structural state assessment. More precisely, the typically considered laws, namely Fick's second law of diffusion (31, 32]), double Fick's law or Langmuirtype law ([16, 33, 34, 35]) for gravimetric approach, completed by Brasher and Kingsbury method 35 for the link with dielectric aspects (36]) - whose origin lies in permittivity approximation in a complex medium (37]) -, usually consider the material as a whole, and only relates the global time-domain evolution (i.e., not assessing the water penetration spatial profile, which can however be of significant importance for monitoring and prognosis purposes). This also results in a pseudoempirical approach for the relationship between gravimetric and dielectric results, not accounting

40 for the particular interactions between a diluted phase (water containing epoxy parts) in a matrix (polymer coating).

Hence, the purpose of this study is twofold. First, it aims at rehabilitating the introduction of spatial variable in the water uptake evolution (also allowing the assessment of the penetration depth) using proper shape functions, and analyzing the obtained theoretical predictions with re-

45 spect to water mass uptake from gravimetric measurements. Secondly, based on this approach, the application of mixing rules between two phases with different permittivities is proposed, allowing the definition of a spatially-distributed permittivity, ultimately giving the global capacitance (or 
equivalent permittivity) of the whole sample. Such an approach therefore permits highlighting possible underlying mechanisms of water uptake and associated measurement techniques, as well

50 as assessing the spatial degradation status of the coating and thus the risk for the coated structure.

The paper is organized as follows. Section 2 exposes the material elaboration procedures as well as gravimetric and dielectric measurement protocols and results. Theoretical model for gravimetry and comparative result analysis with respect to experimental data are then presented in Section 3 Based on this theory, the theoretical link with dielectric aspects, along with the comparison with ${ }_{55}$ experiments, is then presented in Section 4 . Finally, Section 5 recalls the main findings of this study and provide possible future developments and prospects taking advantage of the proposed approach.

\section{Experimental Procedure and Characterization}

The considered material consists in an epoxy-amine network based on BADGE (Bisphenol ${ }_{60}$ A Diglycidyl ether) EPIKOTE ${ }^{\mathrm{TM}}$ Resin 828 cured with polyether amine Jeffamine ${ }^{\circledR}$ D-400 as hardener. In a reactor, BADGE was poured and D-400 agent added at the stoichiometric ratio $(r=1)$. The blend was mechanically stirred before being immersed in an ultrasonic bath in order to remove air bubbles. Then, the mixture was poured in circular shaped silicon molds of $40 \mathrm{~mm}$ in diameter and $2 \mathrm{~mm}$ in thickness. Heat treatment was performed in an electric furnace with the 65 following temperature profile for crosslinking purposes: i) 2 hours at $80^{\circ} \mathrm{C}$, ii) 3 hours at $120^{\circ} \mathrm{C}$, iii) 1 hour at $200^{\circ} \mathrm{C}$ and iv) cooling down at room temperature during 2 hours. Finally, a three step polishing with SiC Emery papers with respective grades of 800, 1500 and 2000 was performed before cutting the coupon in a $30 \mathrm{~mm}$ diameter circular shape (Figure 1(a)). Measured sample thickness was found to be $1.12 \mathrm{~mm}$ in average.

The free-standing sample was then immersed in a saline solution made of water with 0.1 mol. $1^{-1}$ concentration of $\mathrm{NaCl}$ (Figure 1(b) maintained at constant temperature of $35^{\circ} \mathrm{C}$ using a DX602 drying oven from Yamato Corporation ${ }^{\circledR}$. For gravimetric analysis purpose, the sample was

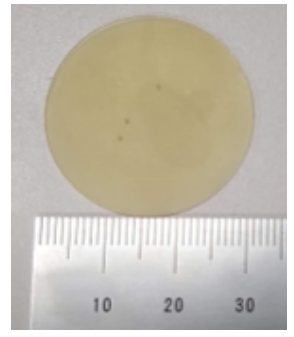

(a) Sample photograph after elaboration

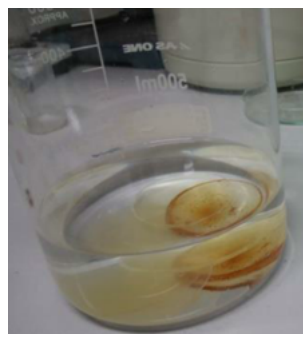

(b) Immersion photograph

Figure 1: Sample photograph and immersion set-up. 
removed from the saline solution at regular time intervals, rinsed to evacuate excess salt contents at the surface, and dried using absorbent paper. The sample mass $M(t)$ was then measured using 75 precision scale As One ${ }^{\circledR}$ Sefi ITX220 with $0.1 \mathrm{mg}$ precision, allowing calculating the mass fraction of water uptake $X_{M}$ as:

$$
X_{M}(\%)=100 \times \frac{M(t)-M_{0}}{M_{0}}
$$

with $M_{0}$ the initial mass $(839.4 \mathrm{mg})$. Results, depicted in Figure 2, shows first a quadratic increase with the time, for eventually reaching a constant value of $2.17 \%(M(\infty)=857.6 \mathrm{mg})$. Such results are consistent with Fick's law as it will be discussed in the next section.

so Following gravimetric analysis, sample was also placed in a Controlled Environment Sample Holder (CESH) from BioLogic ${ }^{\circledR}$ with gold-plated electrodes of $25 \mathrm{~mm}$ in diameter connected to a HP 4194A impedance analyzer for impedance measurement using 4-point probe technique (Figure 3). Capacitance values were then extracted for several frequencies assuming parallel RC model, with results depicted in Figure 4 . This Figure shows a significant dependence of the capacitive part with the immersion time, with a higher sensitivity at low frequencies. Also, a decrease of the capacitance with the frequency can be observed, which is however quite limited (around $-0.5 \mathrm{~dB}$ per decade), but being consistent with other results reported in literature ([20,

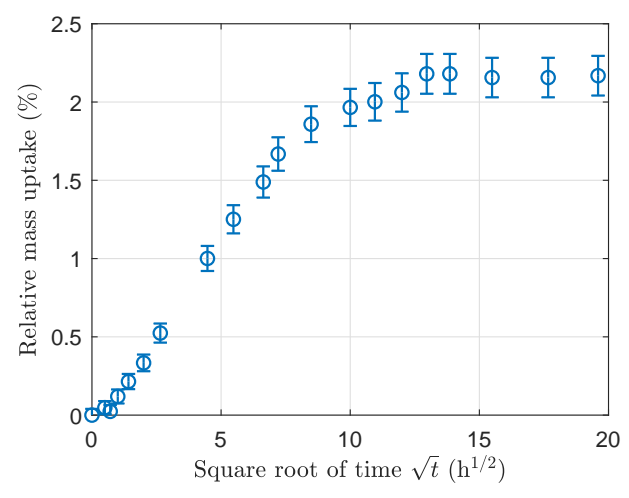

Figure 2: Experimental gravimetric results.

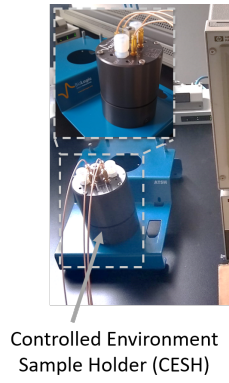

(a) Testbench photograph

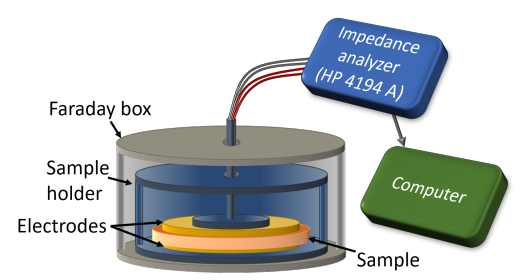

(b) Schematics

Figure 3: Dielectric measurement set-up. 


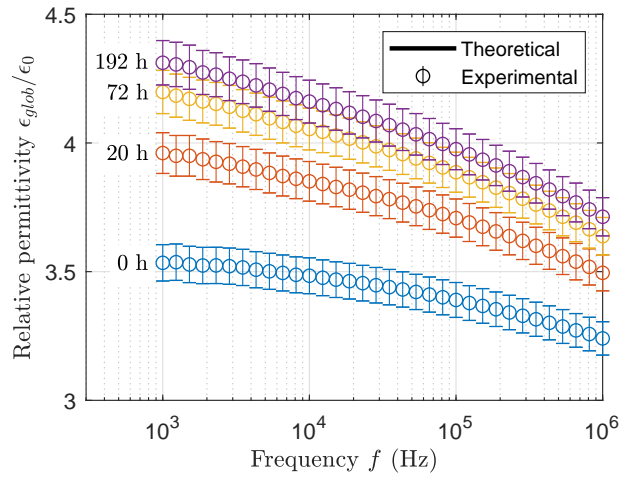

(a) Frequency dependence of capacitance

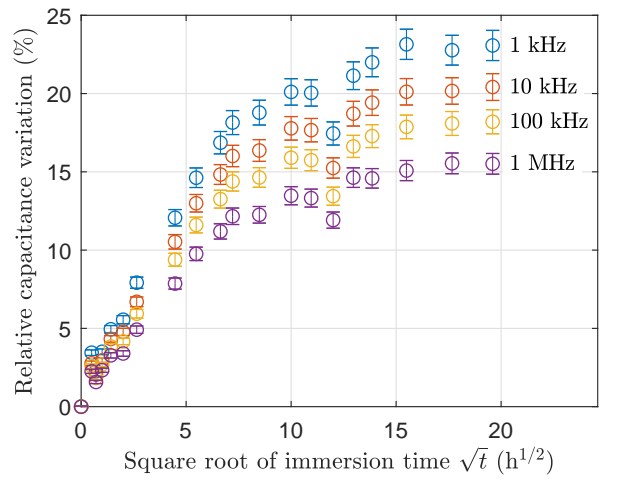

(b) Dependence of relative capacitance variation (with respect to dry capacitance - $t=0 \mathrm{~h}$ ) with immersion time

Figure 4: Experimental dielectric results (time $t=0 \mathrm{~h}$ represents dry coupon).

23]). It can be noted in Figure 4(b) that the point set around $\sqrt{t}=12 \mathrm{~h}^{1 / 2}$ shows a singular value compared to the others (and whatever the considered frequency). While the experimental protocol remained unchanged for all measurements, we attribute this particular value to an external bias (such as momentary bad connection).

\section{Gravimetric Response Modeling and Analysis}

The gravimetric response modeling is usually conducted considering Fick's model at a global scale (i.e., considering the global material), yielding the expression of the time-dependent expression of water absorption ratio (in terms of mass of water uptake), $X_{M}(t)$, as a sum of time-domain relaxation moments, assuming uptake on both sides (i.e., immersed coupon - $[23])^{1}$

$$
\begin{aligned}
X_{M}(t)=\left.X_{M}\right|_{\infty}\left[1-\frac{8}{\pi^{2}} \sum_{j=0}^{\infty}\right. & \frac{1}{(2 j+1)^{2}} \\
& \left.\times \exp \left(-\frac{(2 j+1)^{2} \pi^{2}}{h^{2}} D t\right)\right]
\end{aligned}
$$

where $\left.X_{M}\right|_{\infty}$ is the maximal mass uptake ratio, $h$ the sample thickness, $D$ the diffusion coefficient and $t$ the immersion time. While such a model allows successfully relating the global mass uptake of typical materials (such as amine), it does not permit predicting the effective water penetration within the medium. Hence, in order to be able to assess such a penetration, the genuine Fick's model taking into account the spatial position is considered (39, 40 - assuming unidimensional diffusion), yielding the expression of the mass water absorption ratio density $\chi_{M}(x, t)$ :

\footnotetext{
${ }^{1}$ For single side uptake, the equivalent thickness is simply doubled (24, 38); the diffusion coefficient may change as well.
} 


$$
\begin{aligned}
\chi_{M}(x, t)=\frac{\left.X_{M}\right|_{\infty}}{h}\left\{1-\frac{4}{\pi} \sum_{j=0}^{\infty}\right. & \frac{1}{(2 j+1)} s_{j}(x) \\
& \left.\times \exp \left(-\frac{(2 j+1)^{2} \pi^{2}}{h^{2}} D t\right)\right\}
\end{aligned}
$$

with $x$ the spatial position $(0 \leq x \leq h)$ and $s_{j}(x)$ a shape function sequence defining the spatial distribution of the water uptake within the coating, which verifies Fick's second law conditions (assuming constant concentration) applied to water absorption ratio density:

$$
\nabla^{2} \chi_{M}(x, t)=0
$$

as well as:

$$
\left\{\begin{array}{l}
s_{j}(0)=0 \\
s_{j}(h)=0 \\
s_{j}(x) \geq 0 \forall x \in[0, h] \\
\int_{0}^{h} s_{j}(x) d x=\frac{2}{\pi(2 j+1)} h
\end{array}\right.
$$

Note that in the case of single side uptake, the second condition turns to $s_{j}(h)=1$. Then, the global mass uptake $X_{M}(t)$ can be found through integration of Eq. (3):

$$
X_{M}(t)=\int_{0}^{h} \chi_{M}(x, t) d x
$$

which is actually strictly equivalent to Eq. (2) as space and time are independent variables.

Assuming that the diffusion coefficient is constant and the space and time variables are separable, it can be shown that the shape function is a sine with $n+1 / 2\left(n \in \mathbb{N}^{+}\right)$periods $([39,40])^{2}$

$$
s_{j}(x)=\sin \left[(2 j+1) \pi \frac{x}{h}\right]
$$

Applying such a model using parameters listed in Table 1 yields the result depicted in Figure 5 . along with the comparison with experimental measurements and classical time-domain only Fick's law. Model parameters were obtained from direct measurement for the relative maximal mass

\begin{tabular}{lc} 
Parameter & Value \\
\hline Relative maximal mass & $2.17 \%$ \\
uptake ratio $\left.X_{M}\right|_{\infty}$ & \\
Diffusion coefficient $D$ & $8 \times 10^{-9} \mathrm{~cm}^{2} \cdot \mathrm{s}^{-1}$ \\
\hline
\end{tabular}

Table 1: Parameters for gravimetric analysis.

\footnotetext{
${ }^{2}$ In single side uptake a sine with with $n / 2+1 / 4\left(n \in \mathbb{N}^{+}\right)$periods can be also considered.
} 


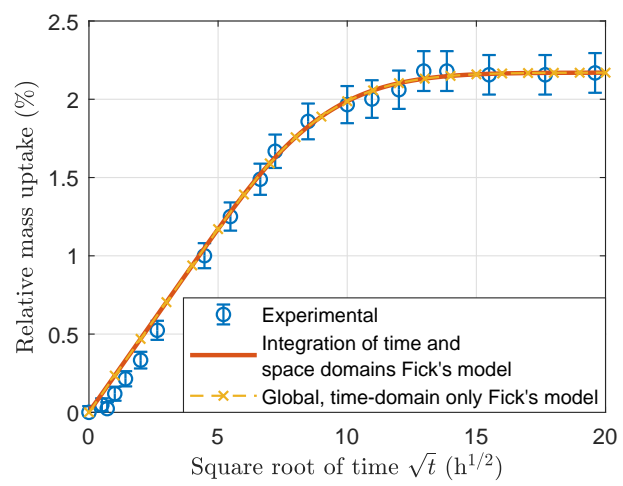

Figure 5: Gravimetric model results and comparison with experiment.

uptake ratio $\left.X_{M}\right|_{\infty}$ (as it corresponds to the saturated value), and from fitting procedure regarding the diffusion coefficient $D$. Results thus demonstrate quite good agreement with experimental values, showing first a linear increase of the relative water mass uptake with the square root of immersion time, and eventually reaching a saturating value at $\left.X_{M}\right|_{\infty}$. Furthermore, a perfect matching can be observed between the integration of the local Fick's law with the conventional global one, thanks to a shape function $s_{j}(x)$ verifying the conditions defined in Eq. (5), validating the relevance of the proposed approach. It can be noted that, because of the definition of the shape function itself, (Eq. (5)), this equivalence between the time-dependent and time- and spacedependent Fick's laws does not allow assessing the validity of the sine shape function defined in Eq. (7) at this stage.

However, while in a global point of view both integrated local or global Fick's laws can be considered, the former yields the significant advantage of assessing the actual water penetration. To this end, Figure 6 shows the time and space distribution of the normalized mass uptake (with respect to its maximal value). In the case of double side uptake such as in the present case of epoxy coupons, this allows evaluating the penetration depth of the water. As an example, it can be inferred that as long as the immersion time is less than approximately $6 \mathrm{~h}$, almost no water has reached the middle of the coupon, while, after approximately $40 \mathrm{~h}$ of immersion, the relative mass uptake density on this surface is almost $50 \%$ of its maximal value (Figure 6(b)). In an applicative point of view (where single side uptake occurs), such results have significant interest to assess the fact that water did reached or not the other surface of the coated material.

\section{Dielectric Response Analysis and Interpretation in Regards to Gravimetric Results}

The gravimetric investigations allowed unveiling new insights in the prognosis of the coating by consideration of the spatial distribution of the uptake. To complete this approach in the framework of in-situ techniques, this section proposes to assess the dielectric measurement results using a different approach than the conventionally used Brasher and Kingsbury model ([36]), taking advantage of the spatial formulation. 


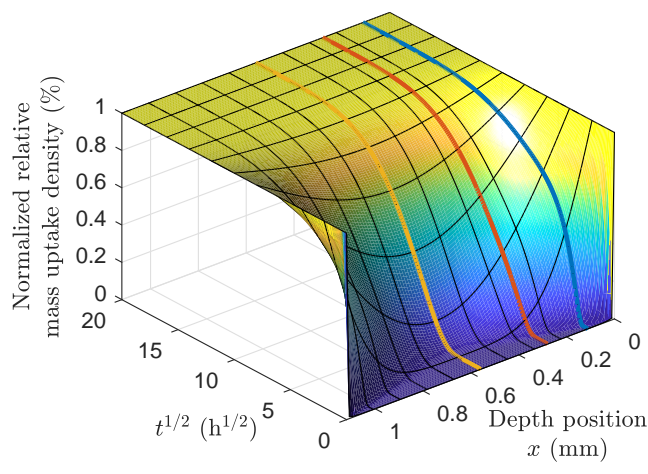

(a) Global view (highlighted curves corresponds to 1/10 (blue), 1/4 (red) and 1/2 (yellow) of the coupon thickness, in accordance with Figure 6(b)

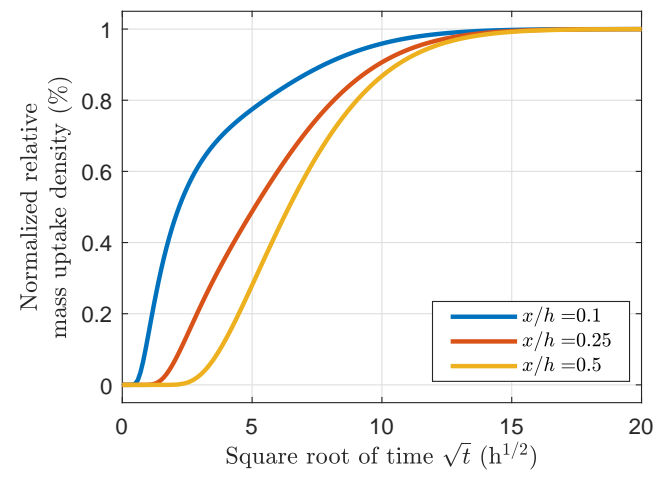

(b) Water penetration at $1 / 10$, quarter and middle of coupon thickness

Figure 6: Mass uptake penetration as a function of immersion time.

More precisely, considering that the water uptake leads to the presence of a secondary phase in the coating, it is possible to consider the local permittivity $\epsilon$ of the full composite (coating + secondary phase) at a specific depth $x$ defined by standard mixing rules. Note that the secondary phase may not be water only due to the interaction and reaction between water and polymer (e.g., polymer degradation), yielding a mixture of water and epoxy that has its own dielectric properties. Denoting $\delta(x, t)$ the time and space-dependent volume phase ratio of secondary phase, and considering that water penetration forms paths in the coating so that each infinitesimal slice of coating leads to a homogeneous distribution of the phases (Figure 7), an associated local permittivity given by 1-3 composite in parallel connection (41]) mixing law can be obtained as:

$$
\epsilon(x, t, \omega)=\delta(x, t) \epsilon_{\text {sec }}(\omega)+[1-\delta(x, t)] \epsilon_{\text {coat }}(\omega)
$$

where $\epsilon_{\text {coat }}(\omega)$ and $\epsilon_{\text {sec }}(\omega)$ are the frequency-depend permittivities of the coating material and secondary phase induced by water uptake, respectively, with $\omega$ denoting the angular frequency.

While the present model principle is not limited to this assumption, it will be considered in the following that the coating and secondary phase permittivities follow a single phase Cole-Cole equation $([42,43])$ :

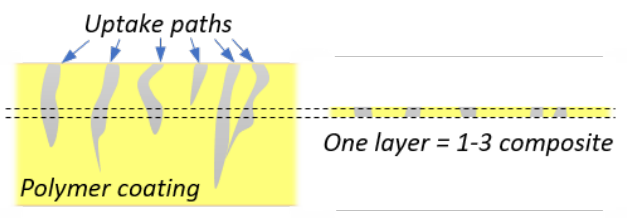

Figure 7: Modeling of each coating layer as 3-1 composite with parallel connection (41]). 


$$
\epsilon_{i}(\omega)=\mathbb{R}\left[\frac{\left.\epsilon_{i}\right|_{\text {init }}-\left.\epsilon_{i}\right|_{\infty}}{1+\left(j \frac{\omega}{\omega_{i}}\right)^{1-\alpha_{i}}}+\left.\epsilon_{i}\right|_{\infty}\right], i=\{\text { coat }, \text { sec }\}
$$

with $\left.\epsilon_{i}\right|_{\text {init }},\left.\epsilon_{i}\right|_{\infty}, \omega_{i}$ and $\alpha_{i}(i=\{c o a t, s e c\})$ respectively defined as the initial (low frequency) permittivity, final (high frequency) permittivity, cut-off angular frequency and Cole-Cole coefficient. $\mathbb{R}$ refers to the real part of the expression.

Based on the previous developments, the phase ratio $\delta(x, t)$ can be defined in a similar way than the water mass uptake, with strictly the same diffusion coefficient $D$, as:

$$
\begin{aligned}
\delta(x, t)=\delta_{\infty} \times\left\{1-\frac{4}{\pi} \sum_{j=0}^{\infty}\right. & \frac{1}{(2 j+1)} s_{j}(x) \\
& \left.\times \exp \left[-\frac{(2 j+1)^{2} \pi^{2}}{h^{2}} D t\right]\right\}
\end{aligned}
$$

where $\delta_{\infty}$ refers to the maximum secondary volume phase ratio (i.e., at saturation of water). Specifically, assuming constant polymer mass and constant coating volume, it is possible to link $\delta_{\infty}$ with the maximal relative mass uptake $\left.X_{M}\right|_{\infty}$ as:

$$
\frac{1}{\delta_{\infty}}=1+\frac{\rho_{w a t}}{\rho_{\text {coat }}} \frac{1}{\left.X_{M}\right|_{\infty}}
$$

where $\rho_{\text {wat }}$ denotes the water density and $\rho_{\text {coat }}$ the (pristine) coating density.

Following this space and immersion time-dependent definition, the so-obtained composite local permittivity as a function of the depth and immersion time can be obtained following Eq. (8). Nevertheless, the capacitive measurement giving a global response of the total coupon, it is possible to extract an equivalent permittivity $\epsilon_{e q u}(t, \omega)$ of the whole sample as:

$$
\epsilon_{\text {equ }}(t, \omega)=\left(\frac{1}{h} \int_{0}^{h} \frac{1}{\epsilon(x, t, \omega)} d x\right)^{-1}
$$

Using previous parameters from gravimetric analysis (diffusion coefficient $D$ and relative maximal mass uptake ratio $\left.X_{M}\right|_{\infty}$ - Table 1 as well as additional values listed in Table $2 \mathbb{\beta}$ leads to results depicted in Figure 8, where the experimental equivalent permittivities have been obtained by multiplying the measured capacitance by the mean sample thickness and inverse electrode surface, yielding polymer coating equivalent permittivity in the same range than reported values in the literature $(44,45])$. Cole-Cole parameters where obtained considering both the slope (using logarithmic x-scale) and curvature of the experimental charts, while the maximal volume phase ratio has been calculated using Eq. 111 from the pristine coating and saline solution densities, yielding a value of $\delta_{\infty}=2.16 \%$. It can be noted that the Cole-Cole coefficients are quite high,

\footnotetext{
${ }^{3}$ Note that for single frequency assessment, only three parameters are needed: the permittivity of each phase (coating and secondary phase) and solution to coating density ratio.
} 


\section{Coating material parameters}

\begin{tabular}{ll} 
Low-frequency permittivity $\left.\epsilon_{\text {coat }}\right|_{\text {init }}$ & $3.57 \epsilon_{0}$ \\
High-frequency permittivity $\left.\epsilon_{\text {coat }}\right|_{\infty}$ & $2.98 \epsilon_{0}$ \\
Cut-off angular frequency $\omega_{\text {coat }}$ & $1.3 \pi \times 10^{6}{\mathrm{rad} . \mathrm{s}^{-1}}$ \\
Cole-Cole coefficient $\alpha_{\text {coat }}$ & 0.6 \\
Density $\rho_{\text {coat }}$ & $1060 \mathrm{~kg} . \mathrm{m}^{-3}$ \\
\hline
\end{tabular}

Saline solution parameters

Density $\rho_{\text {wat }} \quad 1040 \mathrm{~kg} \cdot \mathrm{m}^{-3}$

Secondary phase parameters

Low-frequency permittivity $\left.\epsilon_{\text {sec }}\right|_{\text {init }} \quad 80 \epsilon_{0}$

High-frequency permittivity $\left.\epsilon_{\text {sec }}\right|_{\infty} \quad 12 \epsilon_{0}$

Cut-off angular frequency $\omega_{\text {sec }} \quad 200 \pi$ rad.s ${ }^{-1}$

Cole-Cole coefficient $\alpha_{\text {sec }} \quad 0.87$

Table 2: Additional parameters for dielectric analysis ( $\epsilon_{0}$ denotes the vacuum permittivity).

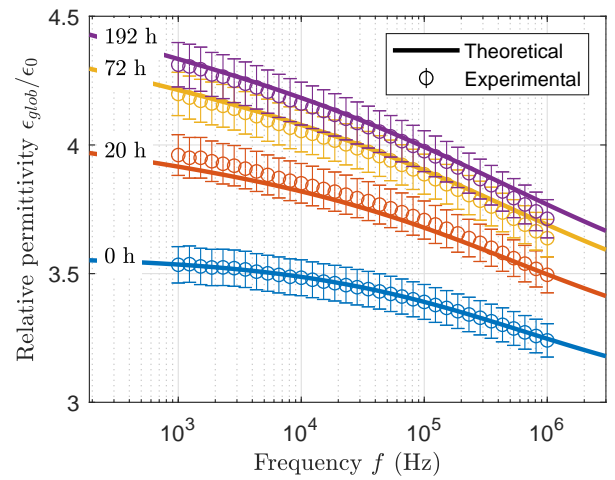

(a) Frequency responses for several immersion time periods

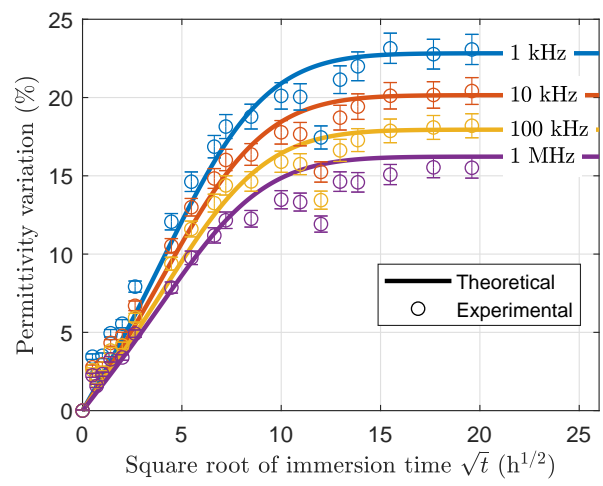

(b) Time responses for several frequencies

Figure 8: Dielectric model results and comparison with experiments (time $t=0 \mathrm{~h}$ represents dry coupon). 
due to the limited decreasing slope with frequency (consistent with the literature - [23, 20]), but it should be kept in mind that the use of such a permittivity model does not have any impact on the exposed principles and can be advantageously replaced by another one (note that the same observation also applies for the mixing rule or for the evolution of the water mass uptake; the latter being possibly non-Fickian).

Still, Figure 8 demonstrates that the proposed approach, providing a physically-based model taking advantage of time and space distributions of the water uptake together with mixing rule theory, allows well predicting the dielectric measurements using results from gravimetry and thus offers a robust method for interpreting measured capacitance and associated variations while giving it physical significance, although some slight permittivity underestimation and overestimation can be observed in medium and high immersion time periods, respectively (and not taking into account the singular points at $\sqrt{t}=12 \mathrm{~h}^{1 / 2}$ as they arise from external measurement issues). The model therefore allows explaining both qualitatively and quantitatively the highest sensitivity at low frequency, through the use of the Cole-Cole transition model which relates the decrease of secondary phase permittivity with frequency, thus decreasing the absolute difference between secondary phase and coating dielectric parameters. According to Figure 8(b), the sensitivity for the maximal immersion time goes from $22.8 \%$ to $16.2 \%$ theoretically when changing the frequency from $1 \mathrm{kHz}$ to $1 \mathrm{MHz}$, which corresponds well to the experimental values of $23.1 \%$ and $15.5 \%$, respectively. These results also give, to some extent, the confirmation of the space distribution assumption of the diffusion (Eq. (7)), as the equivalent permittivity (Eq. (12) ) is strongly dependent on the spatial shape of diffusion as per Eq. 10. Indeed, a badly chosen shape function would yield a totally different spatial distribution of the phase phase and hence local permittivity, leading to dramatic changes in the final equivalent permittivity.

\section{Conclusion}

This study reported an original approach for assessing the water uptake of epoxy coating for anti-corrosion purpose, along with associated measurement technique for its assessment. More specifically, the originality of the proposed method lies in considering the space dependence of the uptake through a presupposed shape function, combined with the application of mixing rules for the assessment of dielectric measurements. In addition of providing an alternative physical explanation of underlying mechanisms of water uptake and associated measurements (gravimetry and dielectric) as well as links between them, such a model also permits estimating the actual water penetration in the coating. The correlation with global measurements, both in terms of water mass uptake and dielectric capacitance, shows very good agreement, giving a relevant assessment of time-dependency of spatial penetration from global measurements.

The identification procedure, highlighting the interdependence of gravimetric and dielectric measurements, can be summarized as follows: 
1. Gravimetric measurement

(a) Determination of the relative maximal mass uptake ratio $\left.X_{M}\right|_{\infty}$ (for sufficiently high equivalent immersion time to reach saturation).

(b) Determination of the diffusion coefficient $D$ from time-dependent relative mass uptake (e.g., fitting).

2. Dielectric measurement (time-dependence is obtained through the diffusion coefficient $D$ calculated from gravimetry).

(a) Calculation of the maximum secondary volume phase ratio $\delta_{\infty}$ from the phase mass density ratio and $\left.X_{M}\right|_{\infty}$ (Eq. [11) ).

(b) Determination of coating permittivity from pristine case capacitance measurement.

(c) Determination of secondary phase permittivity (for sufficiently high equivalent immersion time to reach saturation and using $\delta_{\infty}$ and pristine coating permittivity) through inversion of mixing law.

Further works may include modeling aspects, including for example non-Fickian behavior or including a three-phase model, as well as applicative insights, through the development of an inverse approach (from capacitance measurement to space and time uptake estimation) for monitoring and prognosis technique. Experimental investigation of spatial uptake profile is also to be considered to provide direct assessment and validation regarding the shape function (that has only be validated by indirect measurement through permittivity in the present work).

\section{Acknowledgements}

This work has been performed in the framework of the POMADE project supported by ELyT Global LIA and IFS Lyon Center, and has been partly funded under the Collaborative Research Project of Lyon Center, IFS, Tohoku University (grant number J19Ly02).

L. Ollivier-Lamarque gratefully acknowledges the support of Kamei foundation. M. Lallart and H. Miki gratefully acknowledge the support of JSPS through invitational fellowship grant number L19530, as well as INSA-Lyon for its support through the CRCT program.

\section{Data Availability}

The raw/processed data required to reproduce these findings cannot be shared at this time as the data also forms part of an ongoing study. 


\section{References}

[1] G. H. Koch, M. P.H. Brongers, N. G. Thompson, Y. P. Virmani and J. H. Payer, "Corrosion Cost and Preventative Strategies in the United States", Report No. FHWA-RD-01-156, 773, NACE International, Houston, 2002.

[2] Z. Ahmad, "Principles of Corrosion Engineering and Corrosion Control - 1st Edition", Butterworth-Heinemann pub., 2006. ISBN: 9780750659246 (paper) / 9780080480336 (eBook)

[3] E. Ghali, V. S. Sastri, and M. Elboujdaini, "Corrosion Prevention and Protection: Practical Solutions", John Wiley \& Sons Pub., 2007. ISBN: 978-0-470-02402-7

[4] G. K. van der Wel, O.C.G. Adan, "Moisture in organic coatings - a review", Progr. Orga. Coatings, Vol. 37, pp. 1-14, 1999.

[5] P. Sørensen, S. Kiil, K. Dam-Johansen, C. Weinell, "Anticorrosive coatings: a review", J. Coatings Tech. Res., Vol. 6, pp. 135-176, 2009.

[6] S.V. Gnedenkov, S.L. Sinebryukhov, D.V. Mashtalyar, V.S. Egorkin, M.V. Sidorova and S. Gnedenkov, "Composite polymer-containing protective coatings on magnesium alloy MA8", Corr. Sci., Vol. 85, pp. 52-59, 2014.

[7] K. Qi, Y. Sun, H. Duan and X. Guo, "A corrosion-protective coating based on a solutionprocessable polymer-grafted graphene oxide nanocomposite", Corr. Sci., Vol. 98, pp. 500-506, 2015.

[8] F. Deflorian, S. Rossi, L. Fedrizzi, C. Zanella, "Comparison of organic coating accelerated tests and natural weathering considering meteorological data", Progr. Orga. Coatings, Vol. 59; pp. 244-250, 2007.

[9] S. G. Croll, X. Shi, B. M. D. Fernando, "The interplay of physical aging and degradation during weathering for two crosslinked coatings", Progr. Orga. Coatings, Vol. 61, pp. 136-144, 2008.

[10] M. A. Machado, K.-N. Antin, L. S. Rosado, P Vilaça and T. G. Santos, "Contactless highspeed eddy current inspection of unidirectional carbon fiber reinforced polymer", Composites Part B, Vol. 168, pp. 226-235, 2019.

[11] Y. Zhao, L. Lin, X.M. Li and M.K. Lei, "Simultaneous determination of the coating thickness and its longitudinal velocity by ultrasonic nondestructive method", NDT and E Int., Vol. 43, pp. 579-585, 2010.

[12] A. Mazzinghi, A. Freni and L. Capineri, "A microwave non-destructive testing method for controlling polymeric coating of metal layers in industrial products", NDT and E Int., Vol. 102, pp. 207-217, 2019. 
[24] F. E. Bedoya-Lora, F. Echeverría and J. A. Calderón, "Effectiveness of non-Fickian diffusion model on the water uptake determination of different organic coatings", Progr. Orga. Coatings, Vol. 136, \#105206, 2019. coating thickness on the in-line monitoring of the conversion of photopolymerized acrylate coatings by near-infrared reflection spectroscopy", Polymer, Vol. 50, pp. 1895-1900, 2009.

[14] D. Kranbuehl, D. Hood, J. Rogozinski, A. Meyer and M. Neag, "Monitoring the changing state of a polymeric coating resin during synthesis, cure and use", Progr. Orga. Coatings, Vol. 35, pp. 101-107, 1999.

[15] T. Scherzer, S. Müller, R. Mehnert, A. Volland and H. Lucht, "In-line monitoring of the conversion in photopolymerized acrylate coatings on polymer foils using NIR spectroscopy", Polymer, Vol. 46, pp. 7072-7081, 2005.

[16] W. Liu, S. V. Hoa and M. Pugh, "Water uptake of epoxy-clay nanocomposites: Model development", Comp. Sci. Tech., Vol. 67, pp. 3308-3315, 2007.

[17] W. Liu, S. V. Hoa and M. Pugh, "Water uptake of epoxy-clay nanocomposites: Experiments and model validation", Comp. Sci. Tech., Vol. 68, pp. 2066-2072, 2008.

[18] E. P. M. van Westing, G. M. Ferrari and J. H. W. de Wit, "The Determination of Coating Performance with Impedance Measurements - I. Coating Polymer Properties", Corr. Sci., Vol. 34(9), pp. 1511-1530, 1993.

[19] E. P. M. van Westing, G. M. Ferrari and J. H. W. de Wit, "The Determination of Coating Performance with Impedance Measurements - II. Water Uptake of Coatings", Corr. Sci., Vol. 36(6), pp. 957-977, 1994.

[20] S. Li, L. Liang, J. Li, N.-J. Liu, M. A. Alim, "Characterization of water absorbed epoxy insulating coating material used in $\mathrm{ZnO}$ varistors by dielectric measurements", Mat. Lett., Vol. 60, pp. $114-119,2006$.

[21] C. Corfias, N. Pébère, C. Lacabanne, "Characterization of protective coatings by electrochemical impedance spectroscopy and a thermostimulated current method: Influence of the polymer binder", Corr. Sci., Vol. 42, pp. 1337-1350, 2000.

[22] X. Zhang, F. Wang and Y. Du, "Protective performance of epoxy resin modified with coal tar coating studied by electrochemical impedance spectroscopy", Progr. Orga. Coatings, Vol. 53, pp. 302-305, 2005.

[23] A. N. Fraga, E. Frullloni, O. de la Osa, J.M. Kenny and A. V'azquez, "Relationship between water absorption and dielectric behaviour of natural fibre composite materials", Polymer Testing, Vol. 25, pp. 181-187, 2006. 
[25] T. Nguyen, D. Bentz, E. Byrd, "Method for measuring water diffusion in a coating applied to a substrate", J. Coat. Technol. Res., Vol. 67, pp. 37-46, 1995.

[26] M. C. Santos, B. Bendiksen, Y.A. Elabd, "Diffusion of liquid water in free-standing polymer films using pressure-contact time-resolved fourier transform infrared attenuated total reflectance spectroscopy", Ind. Eng. Chem. Res., Vol. 56, pp. 3464-3476, 2017.

[27] S. Morsch, S. Lyon, P. Greensmith, S.D. Smith, S.R. Gibbon, "Mapping water uptake in organic coatings using AFM-IR", Faraday Discuss., Vol. 180, pp. 527-542, 2015.

[28] M. Arhant, P.-Y. Le Gac, M. Le Gall, C. Burtin, C. Briançon, P. Davies, "Modelling the non Fickian water absorption in polyamide 6", Polym. Degrad. Stab., Vol. 133, pp. 404-412, 2016 .

[29] C. Vosgien Lacombre, G. Bouvet, D. Trinh, S. Mallarino and S. Touzain, "Water uptake in free films and coatings using the Brasher and Kingsbury equation: a possible explanation of the different values obtained by electrochemical Impedance spectroscopy and gravimetry", Electrochimica Acta, Vol. 231, pp. 162-170, 2017.

[30] V. N. Nguyen, F. X. Perrin and J. L. Vernet, "Water permeability of organic/inorganic hybrid coatings prepared by sol-gel method: a comparison between gravimetric and capacitance measurements and evaluation of non-Fickian sorption models", Corr. Sci., Vol. 47, pp. 397$412,2005$.

[31] A. Fick, "On liquid diffusion", Annalen Phys. Chem., Vol. 94, pp. 59-86, 1855.

[32] A. Fick, "On liquid diffusion", J. Memb. Sci., Vol. 100, pp. 33-38, 1995.

[33] I. Langmuir, "The Adsorption of Gases on Plane Surface of Glass, Mica and Platinum", Res. Lab. General Electric Co., Vol. 40(9), pp. 1361-1402, 1918.

[34] H. G. Carter and K. G. Kibler, "Langmuir-type model for anomalous moisture diffusion in composite resins", J. Comp. Mater., Vol. 12, pp. 118-131, 1978.

[35] M. E. Gurtin and C. Yatomi, "On a model for two phase diffusion in composite materials", J. Comp. Mater., Vol. 13, pp. 126-130, 1979.

[36] D. M. Brasher, A. H. Kingsbury, Electrical measurements in the study of immersed paint coatings on metal. I. Comparison between capacitance and gravimetric methods of estimating water-uptake, Journal of Applied Chemistry, Vol. 4, pp. 62-72, 1954.

[37] L. Hartshorn, N. J. I. Megson, E. Rushton, "The structure and electrical properties of protective films", J. Soc. Chem. Ind., Vol. 56, pp. T266-T270, 1937. 
[38] C.-H. Chen and G. S. Springer, "Moisture Absorption and Desorption of Composite Materials", J. Comp. Mat., Vol. 10, pp. 2-20, 1976.

[39] J. Crank, "The Mathematics of Diffusion, 2nd Ed.", Oxford University Press, Ely House, London W.I, 1975. ISBN: 0-19-853344-6

[40] S. Allen and T. Eagar, "Thermodynamics and Kinetics of Materials", Massachusetts Institute of Technology: MIT OpenCourseWare, 3.205, Fall 2006. https://ocw.mit.edu (last viewed: $01 / 11 / 2019)$

[41] R. E. Newnham, D. P. Skinner and L. E. Cross, "Connectivity And Piezoelectric-Pyroelectric Composites", Mat. Res. Bull., Vol. 13, pp. 525-536, 1978.

[42] K. S. Cole and R. H. Cole, "Dispersion and Absorption in Dielectrics - I Alternating Current Characteristics", J. Chem. Phys., Vol. 9(4), pp. 341-352, 1941.

[43] K. S. Cole and R. H. Cole, "Dispersion and Absorption in Dielectrics - II Direct Current Characteristics", J. Chem. Phys., vol. 10(2), pp. 98-105, 1942.

[44] J. Yu, R. Huo, C. Wu, X. Wu, G. Wang and P. Jiang, "Influence of Interface Structure on Dielectric Properties of Epoxy/Alumina Nanocomposites", Macromol. Res., Vol. 20(8), pp. 816-826, 2012.

[45] I. A. Saeedi, T. Andritsch and A. S. Vaughan, "On the Dielectric Behavior of Amine and Anhydride Cured Epoxy Resins Modified Using Multi-Terminal Epoxy Functional Network Modifier", Polymers, vol. 11, \# 1271, 2019. 